\title{
Interaction between Fusarium oxysporum f. sp. cubense and Radopholus similis can lead to changes in the resistance of banana cultivars to Fusarium wilt
}

\author{
Anelita de Jesus Rocha • Mileide dos Santos Ferreira • Leandro de Souza Rocha • \\ Saulo A. S. Oliveira • Edson Perito Amorim • Eduardo S. G. Mizubuti • Fernando Haddad
}

Accepted: 21 July 2020 / Published online: 3 August 2020

(C) The Author(s) 2020

\begin{abstract}
Fusarium oxysporum f. sp. cubense (Foc) causes Panama disease or Fusarium wilt of bananas. The association between soil-inhabiting fungi and nematodes can increase the severity of symptoms and suppress the resistance of plants to diseases. In this study, the interaction between Foc race 1 and Radopholus similis, a burrowing nematode that parasitizes banana plants, was analyzed using one moderately susceptible cultivar and seven resistant cultivars of banana. Two Foc isolates that differed in virulence were tested. The analyses of symptoms and stained fungal structures in the roots demonstrated that $R$. similis interacting with Foc in different inoculation sequences caused changes in symptom severity and the resistance pattern to Foc isolate 0801 (race 1) in cultivars 'Terra Maranhão', 'BRS Pacovan Ken', 'BRS Vitória', and 'BRS Platina'. The data generated in this study have relevant
\end{abstract}

Electronic supplementary material The online version of this article (https://doi.org/10.1007/s10658-020-02081-y) contains supplementary material, which is available to authorized users.

A. d. J. Rocha $\cdot$ M. d. S. Ferreira

Universidade Federal do Recôncavo da Bahia, Rua Rui Barbosa, 710 - Centro, CEP, Cruz das Almas, Bahia 44380-000, Brazil

L. d. S. Rocha · S. A. S. Oliveira • E. P. Amorim •

F. Haddad $(\bowtie)$

Embrapa Mandioca e Fruticultura, Rua Embrapa, s/n, CP 007, Centro, CEP, Cruz das Almas, Bahia 44380-000, Brazil

e-mail: fernando.haddad@embrapa.br

E. S. G. Mizubuti

Universidade Federal de Viçosa, Av. Peter Henry Rolfs, s/n Campus Universitário, Viçosa, Minas Gerais, Brazil implications for banana breeding programs in the classification of cultivars for durable resistance to Fusarium wilt and for understanding pathogen interactions during occurrence of the disease.

Keywords Banana $\cdot$ Fungi $\cdot$ Phytonematodes $\cdot$ Musa . Synergism

\section{Introduction}

Fusarium oxysporum f. sp. cubense (EF Smith) Snyder and Hansen (Foc) causes Panama disease or Fusarium wilt (FW) in bananas, a fungal disease that is a serious threat to both export crops and small-scale banana production worldwide (Ploetz, 2015). Another pathogen of fruit crops, including bananas, is the burrowing nematode Radopholus similis (Cobb) Thorne. Overall, $R$. similis is considered the fourth most destructive nematode that attacks different crops and the most damaging to banana cultivation (Hartman et al. 2010; Jones et al. 2013; Sankar et al. 2017).

Foc is a soil necrotrophic pathogen that produces chlamydospores, survival spores that remain viable allowing the disease to persist and preventing the use of susceptible banana cultivars. Consequently, the use of resistant cultivars is the most efficient way to control FW (Li et al. 2013; Costa et al. 2015; Haddad et al. 2018). Banana genotypes have been evaluated via the banana breeding program of Embrapa in Brazil to detect genotypes resistant to the pathogens among diploid accessions with an appropriate level of genetic 
variability, such as M53, Jaran, Birmanie, Pipit, and Malaccensis and between the Figo type and Silver type genotypes with Foc race 1 resistance alleles (Silva et al. 2011; Rebouças et al. 2018; Ribeiro et al. 2018).

However, new pathogen variants outperform current sources of resistance, as observed in 1990 with the emergence of Subtropical Race 4 (STR4) and Tropical Race 4 (TR4); the latter is a highly virulent variant of Foc that decimated plantations of Cavendish bananas in South East Asia (Stover 1962; Ploetz 2006). Foc TR4 is pathogenic under tropical and subtropical conditions, but Foc STR4 is currently known to cause FW only in Cavendish plants in the subtropics (Ploetz 1990; Ploetz 2006; Ghag et al., 2015). TR4 is a constant threat to banana production, and the disease has spread to other countries of Southeast and South Asia, the Middle East, Africa, and South America (Ploetz 2015; Dita et al. 2018; García-Bastidas et al. 2019). One phenotypic trait that distinguishes between STR4 and TR4 is the vegetative compatibility groups (VCGs) to which the variants belong. Among the 24 known VCGs of Foc, VCG 0120, 01201, 01202, 01209, 01210, 01211, 01215, and 0120/15 are associated with Foc STR4, whereas only one VCG, 01213/16, has been reported for Foc TR4 (Buddenhagen 2009; Dita et al. 2018).

In addition to the variants that exist in the Foc population, the interaction of the fungal pathogens with nematodes may interfere with the expression and durability of resistance to FW. The breakdown of resistance is not only related to the damage caused by the nematodes that allow the pathogen to enter the roots, but also to the production and secretion of proteins associated with the pathogenicity that interfere with gene expression and the cell metabolism of the host plant (Davis et al. 2000; Hussey et al. 2002; Davis et al. 2004; Rocha et al. 2018). Previous studies assessed the effects of the interactions between Foc and $R$. similis in banana cultivars using susceptible and moderately susceptible cultivars to FW. The major conclusion was that the interaction was synergistic (Somu et al. 2013; Dinesh et al. 2014). However, the effects of the interaction between Foc and $R$. similis in banana cultivars resistant to FW remains unknown.

In this sense, knowledge of the interactions between Foc and $R$. similis, in the process that leads to FW is necessary to delineate control strategies for the disease. This study tested the following hypotheses: (i) Foc resistance and basal type based on pre-formed barriers can be overcome because of the interaction with
$R$. similis; and (ii) the interaction between a virulent pathotype of Foc and $R$. similis can lead to more severe symptoms of FW in resistant cultivars. To test these hypotheses, the interactions between Foc and $R$. similis were evaluated in banana cultivars resistant to FW.

\section{Materials and methods}

Selecting and multiplying Foc isolates and $R$. similis

The CNPMF 0801 and CNPMF 218A Foc isolates were selected from the biological collection of $F$. oxysporum f. sp. cubense in the phytopathology laboratory at Embrapa. Based on a host range test, the CNPMF 0801 isolate was classified as race 1 and is the standard for the studies. Isolate 218A was first found in the State of São Paulo affecting the cultivar Nanica (Cavendish). In a previous study, the $218 \mathrm{~A}$ isolate was reported as virulent because it caused symptoms of FW in Grand Naine, a Cavendish type cultivar, and was characterized as possibly a new pathotype of Foc in Brazil (Costa et al. 2015; Velame 2017). Thus, VCGs were used to distinguish the 218A isolate between Tropical Race 4 (TR4) and Subtropical Race 4 (STR4). For the induction and recovery of nit mutants, the methodology described by Puhalla (1985) was used.

The culture medium, composed of sand and cornmeal sterilized and incubated at $25{ }^{\circ} \mathrm{C}$ for 15 days, was used to produce mass inocula of Foc, which was quantified by serial dilution and counting colony forming units (CFU) and adjusted to $10^{6} \mathrm{CFU} / \mathrm{g}$ of substrate. The inoculum of $R$. similis was produced in Grande Naine banana seedlings in containers with autoclaved soil. Ninety days after inoculation (DAI), the nematodes were extracted from the roots and soil. A Peters chamber under an optical microscope was used to count the nematodes, and the inoculum was adjusted to 1000 nematodes (females and juveniles)/mL of suspension.

\section{Greenhouse trials}

Two trials were set up in a greenhouse to test the hypotheses of the study from February to June 2017. Based on studies related to $\mathrm{FW}$ resistance, eight banana cultivars were selected (Table 1) (Amorim et al. 2016). The micropropagated seedlings were acclimated and planted in $3 \mathrm{~L}$ containers that contained a mixture of 
commercial substrate based on Pinus bark (Tecnomax $\left.{ }^{\circledR}\right)$ and coconut fiber $(5: 1 ; \mathrm{v}: \mathrm{v})$.

In the first trial, the interaction between CNPMF 0801 and $R$. similis was tested against seven resistant banana cultivars: BRS Japira, BRS Vitória, BRS Platina, BRS Princesa, BRS Pacovan Ken, Grande Naine, and Terra Maranhão. Prata-Anã, a cultivar that is moderately susceptible to FW, was also used. As a conceptual repetition of the first test, in the second trial, the effects of the interactions between pathotype 218A able to infect banana cultivars from the Cavendish group and $R$. similis were assessed. The severity of FW when Foc and $R$. similis were present was assessed using four banana cultivars: BRS Vitória, BRS Pacovan Ken, Grande Naine, and Prata-Anã.

In both trials and for each cultivar, infestation with Foc was conducted by depositing $40 \mathrm{~g}$ of the substrate $\left(10^{6} \mathrm{UFC} / \mathrm{g}\right)$ in four holes (10 cm deep) around the roots in each pot. For the inoculation of $R$. similis, two holes were made in the soil to expose the roots and a suspension of approximately 1000 juvenile and females nematodes was added per pot. The plants used as absolute controls received $40 \mathrm{~g}$ of the sand-cornmeal substrate and were neither inoculated with the fungus nor exposed to the suspension containing nematodes. After inoculation, the plants were kept in a greenhouse where they remained until the end of the evaluations, with temperatures varying between 25 and $30{ }^{\circ} \mathrm{C}$, approximately $60 \%$ relative humidity, and a photoperiod of 12:12 h. The treatments were composed of six different inoculation sequences of Foc and $R$. similis (Table 2). The experimental design was completely randomized. The treatments were applied to nine experimental units (replicates) in the first trial and to seven experimental units in the second trial. An experimental unit consisted of one plant per pot.

Cultivar response in relation to Foc and $R$. similis

The evaluation of the response of the cultivars in relation to FW was assessed based on the expression of external symptoms. Disease intensity was rated using a scale established by Dita et al. (2014): (0) no external symptoms; (1) initial yellowing of old leaves; (2) yellowing of old leaves with initial discoloration of young leaves; (3) all leaves with intense yellowing; and (4) dead plant. Ninety DAI, rhizomes from the plants were cross-cut and evaluated using the scale proposed by Dita et al. (2014):
(0) no symptoms; (1) initial rhizome necrosis; (2) mild necrosis symptoms on the rhizome; (3) intense necrosis symptoms on the rhizome; (4) rhizome with most of the internal tissues showing necrosis; and (5) totally necrotic rhizome.

The obtained scores were converted into a disease index (DI), based on the formula $D I(\%)=100 . \Sigma[(f v) /$ $(n x)]$, where DI is the disease index, $\mathrm{f}$ is the number of plants with the same grade, $v$ is the assigned grade, $n$ is the number of plants evaluated, and $\mathrm{x}$ is the maximum grade from the scale (McKinney, 1923). The area under the disease progress curve (AUDPC) was also calculated based on the development of external symptoms over time, using the formula: $A U D P C=\Sigma\left[\left(\left(y_{1 i}+y_{i+1}\right)\right)^{\prime}\right.$ $\left.2)^{*}\left(t_{i+1}-t_{j}\right)\right]$, where $y_{i}$ is the severity of the disease (based on the DI) in the observed i, $y_{i+1}$ is the severity of the disease at the time of the subsequent evaluation $\mathrm{i}+1$, and $t_{i}$ is the assessment time (days) (Campbell and Madden 1990).

The rhizomes of the plants were evaluated based on the percentage of necrosis using the scale established by Bridge (1988). Necrosis in $<25 \%$ of the rhizome was considered mild, from $25 \%$ to $50 \%$ was moderate, from $51 \%$ to $75 \%$ was severe, and > $75 \%$ was very severe.

The percentage of necrosis in the roots and the number of functional roots were surveyed according to the methodologies described by Speijer and De Waele (1997). After washing, roots were divided into two categories: dead and functional. The number in each category was counted and the value was converted into a percentage. Five $10 \mathrm{~cm}$ long functional primary roots were randomly selected and cut longitudinally. Half of each of the five roots was evaluated to determine the percentage of necrosis. The maximum necrosis per segment of each root was equal to $20 \%$ providing a maximum root necrosis of $100 \%$ for the sum of the five root segments. The sum of the necrosis of each root segment was used as the total root necrosis for the sample (Speijer and De Waele 1997).

The densities of the $R$. similis populations in each treatment were determined by extracting the nematodes from $10 \mathrm{~g}$ of roots and $100 \mathrm{~cm}^{3}$ of soil, based on the methods of Coolen and D'Herde (1972) and Jenkins (1964), respectively. Subsequently, the number of nematodes extracted from the roots and soil of each well was multiplied by root and soil weight and the nematode reproduction factor $(\mathrm{RF}=$ final population/initial population) was calculated. 
Table 1 Banana cultivars used in the study to investigate the effects of interactions between Fusarium oxysporum f. sp. cubense and Radopholus similis on Fusarium wilt. Cruz das Almas, BA

\begin{tabular}{|c|c|c|c|c|c|c|}
\hline Cultivars & Genome & Genealogy (Origin) & Subgroup & Foc $218 \mathrm{~A}$ & Foc 0801 & $R$. similis \\
\hline BRS Japira & $\mathrm{AAAB}$ & Hybrid (Pacovan + M53) & Prata & - & $\mathrm{R}$ & $\mathrm{S}$ \\
\hline Terra Maranhão & $\mathrm{AAB}$ & Cultivar & Plantain & - & $\mathrm{R}$ & $\mathrm{S}$ \\
\hline BRS Platina & $\mathrm{AAAB}$ & Hybrid (Prata-Anã + M53) & Prata & - & $\mathrm{R}$ & $\mathrm{S}$ \\
\hline Prata-Anã & $\mathrm{AAB}$ & Cultivar & Prata & $\mathrm{S}$ & $\mathrm{S}$ & $\mathrm{R}$ \\
\hline Grand Naine & AAA & Cultivar & Cavendish & MR & $\mathrm{R}$ & $\mathrm{S}$ \\
\hline BRS Pacovan Ken & $\mathrm{AAAB}$ & Hybrid (Pacovan + M53) & Prata & $\mathrm{S}$ & $\mathrm{R}$ & MR \\
\hline BRS Princesa & AAAB & Hybrid (Yangambi $\mathrm{n}^{\circ} 2+$ M53) & Silk & - & $\mathrm{R}$ & $\mathrm{S}$ \\
\hline BRS Vitória & AAAB & Hybrid (Pacovan + M53) & Prata & $\mathrm{S}$ & $\mathrm{R}$ & $\mathrm{S}$ \\
\hline
\end{tabular}

$R$ Resistant, $S$ Susceptible, $M R$ Moderately tough, - Not tested

Evaluating the penetration of Foc and $R$. similis

by clearing and staining roots

The procedure for staining the roots was adapted from Phillips and Hayman (1970) and Costa (2013). To clear the roots, samples were cut and placed into containers with a solution of $\mathrm{KOH}(10 \%)$ for $60 \mathrm{~min}$ in a water bath $\left(90{ }^{\circ} \mathrm{C}\right)$. The roots were then acidified in a solution $\mathrm{HCl}$ (1\%) for $20 \mathrm{~min}$ at room temperature. Next, the roots were stained with trypan blue in a $0.05 \%$ solution of lactoglycerol (2:1:1; lactic acid:glycerin:water) and heated for $40 \mathrm{~min}$ in a microwave. The roots were then immersed in a solution of lactoglycerol to remove excess stain and photographed under an optical microscope.

Table 2 Description of treatments applied to evaluate the effects of interactions between Fusarium oxysporum f. sp. cubense and Radopholus similis on the susceptibility of banana cultivars to Fusarium wilt. Cruz das Almas, BA

\begin{tabular}{lc}
\hline Treatments & Acronym \\
\hline Foc inoculated isolated & Foc \\
$\begin{array}{l}\text { Foc inoculated in the planting and R.similis, } \\
\quad \text { inoculated after 7 days }\end{array}$ & Foc $+\mathrm{Rad}$ \\
Foc and $R$. similis, inoculated simultaneously & Foc + Rad \\
& $(\mathrm{S})$ \\
R. similis inoculated at planting, and Foc inoculated & $\mathrm{Rad}+\mathrm{Foc}$ \\
after 7 days & $(7)$ \\
R. similis inoculated isolated & $\mathrm{Rad}$ \\
Non-inoculated control plants & $\mathrm{Control}$ \\
\hline
\end{tabular}

Foc Fusarium oxysporum f. sp. cubense
Data analysis

The data were submitted to a Shapiro and Wilk (1965) normality test. In relation to the percentages of functional roots and root necrosis in the rhizome caused by $R$. similis, the data were arc $\sin \bigvee_{\mathrm{x}} / 100$ transformed. The data regarding the number of nematodes were $\log$ $(x+1)$ transformed. These data were transformed so they conformed to a normal distribution.

The indices of the internal and external symptoms caused by Foc (AUDPC and disease index - DI) and the indices of the $R$. similis (RF, root necrosis, and rhizome necrosis) were analyzed using a heat map constructed with the "gplots" package in the R statistical program (R Core Team 2017). A Pearson correlation analysis was performed between the variables associated with Foc and $R$. similis using the "Performance Analytics" package in $\mathrm{R}$.

\section{Results}

Changes in resistance responses of cultivars

The distributions of internal disease index (IDI) and area under the disease progress curve (AUDPC), stratified by cultivars that were evaluated for isolate 0801 (race 1) and isolate 218A are shown in Fig. 1, and for cultivars evaluated with only isolate 0801 (race 1), in Fig. 2. Both IDI and AUDPC were equal to zero in the cultivar 'Grande Naine' using the isolate of Foc 0801 (race 1), in relation to Foc 218A, IDI and AUDPC had an increase when Foc and $R$. similis were inoculated simultaneously, and when $R$. similis was inoculated 7 days before Foc in "Grande 
Naine" (Fig. 1 and Supplementary Fig. 2). When comparing the virulence profiles (ability to cause disease in a certain variety) of Foc 218A using the host range test, the isolate did not belong to Tropical Race 4 (TR4). This isolate appeared to be STR4 based on tests from the vegetative compatibility group, where it was characterized as belonging to VCG 0120 . The symptoms generated by Foc 218A started 15 DAI and many plants died quickly after that, especially when the Foc was inoculated simultaneously with $R$ similis. In all experiments, it was possible to recover the Foc from the samples and conduct Koch's postulates to confirm the pathogenicity of the isolates.

Regarding Foc 0801, the cultivars 'BRS Pacovan-ken' and 'BRS Vitória' only showed IDI and AUDPC when there were interactions with $R$. similis. In relation to isolate 218A, these cultivars showed IDI and AUDPC in all treatments, without marked differences between them (Fig. 1). The cultivar 'Prata-Anã' seems to have a higher IDI and AUDPC when inoculated with isolate 218A compared to isolate 0801. In both cases IDI and AUDPC increased when Foc and $R$. similis were inoculated simultaneously, and when Foc was inoculated 7 days before R. similis (Fig. 1 and Supplementary Fig. 1).

The cultivars 'BRS Platina' and 'Terra Maranhão' did not show IDI and AUDPC when Foc 0801 (race 1) was inoculated alone (Fig. 2 and supplementary fig. 1). In the case of 'BRS Platina', the average IDI and AUDPC increased when Foc and $R$. similis were inoculated simultaneously (Fig. 2). In relation to 'Terra Maranhão', the mean IDI and AUDPC seem to have an increase when $R$. similis was inoculated 7 days before Foc (Fig. 2). The cultivar 'BRS Japira' showed high IDI in the treatment in which Foc and R. similis were simultaneously inoculated, however the AUDPC does not seem to show great differences between treatments (Fig. 2). In relation to 'BRS Princesa', the average AUDPC and the IDI did not differ in the treatments and the plants exhibited IDI below 10\% (Fig. 2).

\section{Correlation between variables}

In both experiments with different Foc isolates, there were positive correlations between AUDPC and ID, as well as between the variables rhizome necrosis and root necrosis. For isolate 218A the internal index of Foc symptoms was positively correlated with rhizome necrosis (Fig. 3).

A color grouping analysis based on a heat map was performed to classify the cultivars according to resistance (Fig. 4). For the variables associated with Foc (AUDPC and IDI) and the variables associated with $R$. similis (RF, root necrosis, and rhizome necrosis), two groups could be identified in relation to isolates 0801 and 218A (Fig. 4). For both isolates, changes in resistance responses occurred in all treatments with Foc and R. similis (Fig. 4 a).

For AUDPC with isolate 0801, 'BRS Vitória' and 'BRS Pacovan Ken' were classified as resistant when inoculated with Foc alone, but when Foc 0801 was inoculated 7 days before that of $R$. similis, and when the pathogens were simultaneously inoculated both cultivars were classified as moderately resistant (Fig. 4 a). For both AUDPC and IDI, 'Prata-Anã' was classified as susceptible in relation to the isolate Foc 218A. For isolate Foc 0801, a classification as susceptible was possible when assessing the variable IDI (Fig. 4a). In relation to AUDPC, all cultivars were considered susceptible when isolate Foc 218A was singly inoculated, when inoculated with Foc 7 days after that of $R$. similis, or when inoculated with Foc 218A and $R$. similis simultaneously (Fig. 4a).

All cultivars were classified as resistant based on the variables associated with $R$. similis, including rhizome necrosis, root necrosis, and RF, and when $R$. similis was inoculated singly, except for 'BRS Pacovan Ken', and 'Grande Naine', which were classified as susceptible when the reproductive factor was considered (Fig. 4b). 'Prata-Anã' and 'BRS Vitória' inoculated with Foc and $R$. similis simultaneously were considered susceptible based on the reproductive factor of the nematode (Fig. 4b). For 'Grande Naine' and 'BRS Pacovan Ken', the necrosis variable for the rhizome for all the cultivars were considered highly susceptible when $R$. similis was simultaneously inoculated with Foc, whereas 'PrataAnã' and 'BRS Vitória' were classified as moderately resistant to the nematode (Fig. 4b).

Regarding the cultivars evaluated for IDI with Foc 0801 only (Fig. 4c, d), 'Terra Maranhão', and 'BRS Princesa' were considered resistant regardless of the treatment (Fig. 4c). For AUDPC, the cultivars were considered highly susceptible (Fig. 4c). When assessing IDI and AUDPC, 'BRS Platina' was considered resistant when Foc was inoculated singly and when inoculated 7 days after $R$. similis (Fig. 4c). 'BRS Platina' was considered moderately resistant in terms of IDI and AUDPC when both pathogens were simultaneously inoculated (Fig. 4c). 


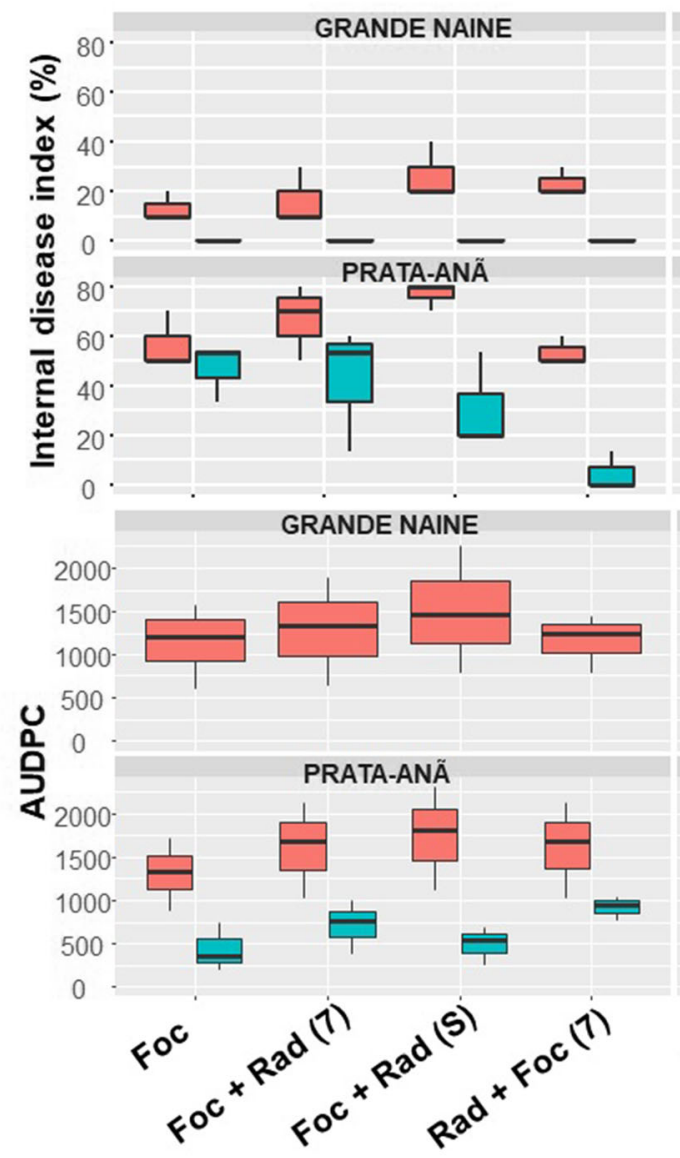

Fig. 1 Effects of the interaction between of two isolates Fusarium oxysporum f. sp. cubense and Radopholus similis in banana cultivars. Foc: Foc inoculated isolated; Foc + Rad (7): Foc inoculated in the planting and $R$. similis inoculated after 7 days; Foc + Rad

For the RF of R. similis, all cultivars were considered susceptible in all treatments, except 'BRS Japira', which was resistant in the treatment in which $R$. similis was inoculated 7 days prior to that of Foc 0801 (Fig. 4d). 'Terra Maranhão' was considered moderately resistant to necrosis of rhizomes and root necrosis, regardless of the inoculation method with Foc 0801 (Fig. 4d). In relation to root necrosis and rhizome necrosis, 'BRS Platina', 'BRS Japira', and 'BRS Princesa' were considered resistant to the nematode regardless of the treatment (Fig. 4d).

\section{Evaluation of functional roots}

The interaction of Foc with $R$. similis reduced the percentage of functional roots in all cultivars compared to that of Foc alone (Fig. 5). In 'Grande Naine', there was a

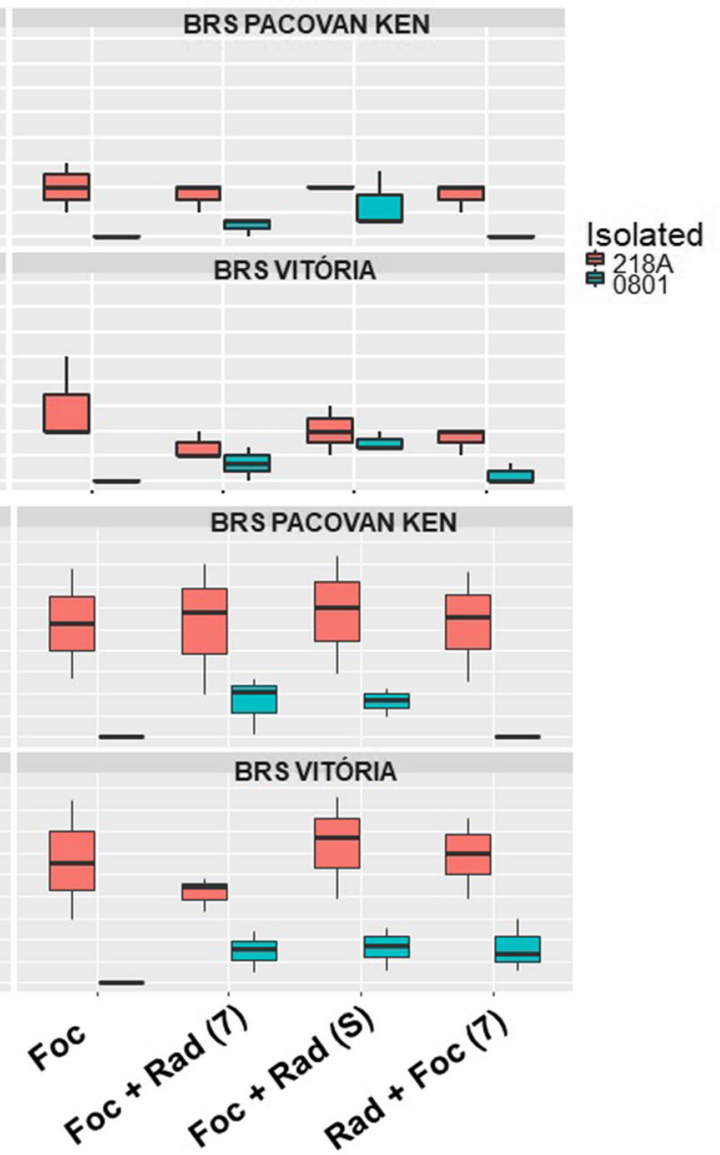

(S): Foc and R. similis inoculated simultaneously; Rad + Foc (7): $R$. similis inoculated in the planting planting and Foc inoculated after 7 days. AUDPC: area under the disease progress curve

greater reduction in the functional roots when $R$. similis was inoculated $7 \mathrm{~d}$ before that of Foc 0801 and the interaction with Foc 218A when the inoculations were simultaneous (Fig. 5). There was a reduction in functional roots in 'Prata-Anã' when Foc was inoculated 7 days before that of $R$. similis and $7 \mathrm{~d}$ later (Fig. 4). As for isolate $218 \mathrm{~A}$, the reduction of functional roots was greater when plants were simultaneously inoculated with Foc and $R$. similis and when $R$. similis was inoculated alone (Fig. 5).

For 'BRS Pacovan Ken', Foc 218A inoculated $7 \mathrm{~d}$ before that of $R$. similis caused a greater reduction in functional roots and the interaction with Foc 0801 did not give significantly greater reductions than did $R$. similis inoculated alone (Fig. 5). For 'BRS Vitória', the treatment in which Foc 218A and $R$. similis were inoculated simultaneously reduced the percentage of 


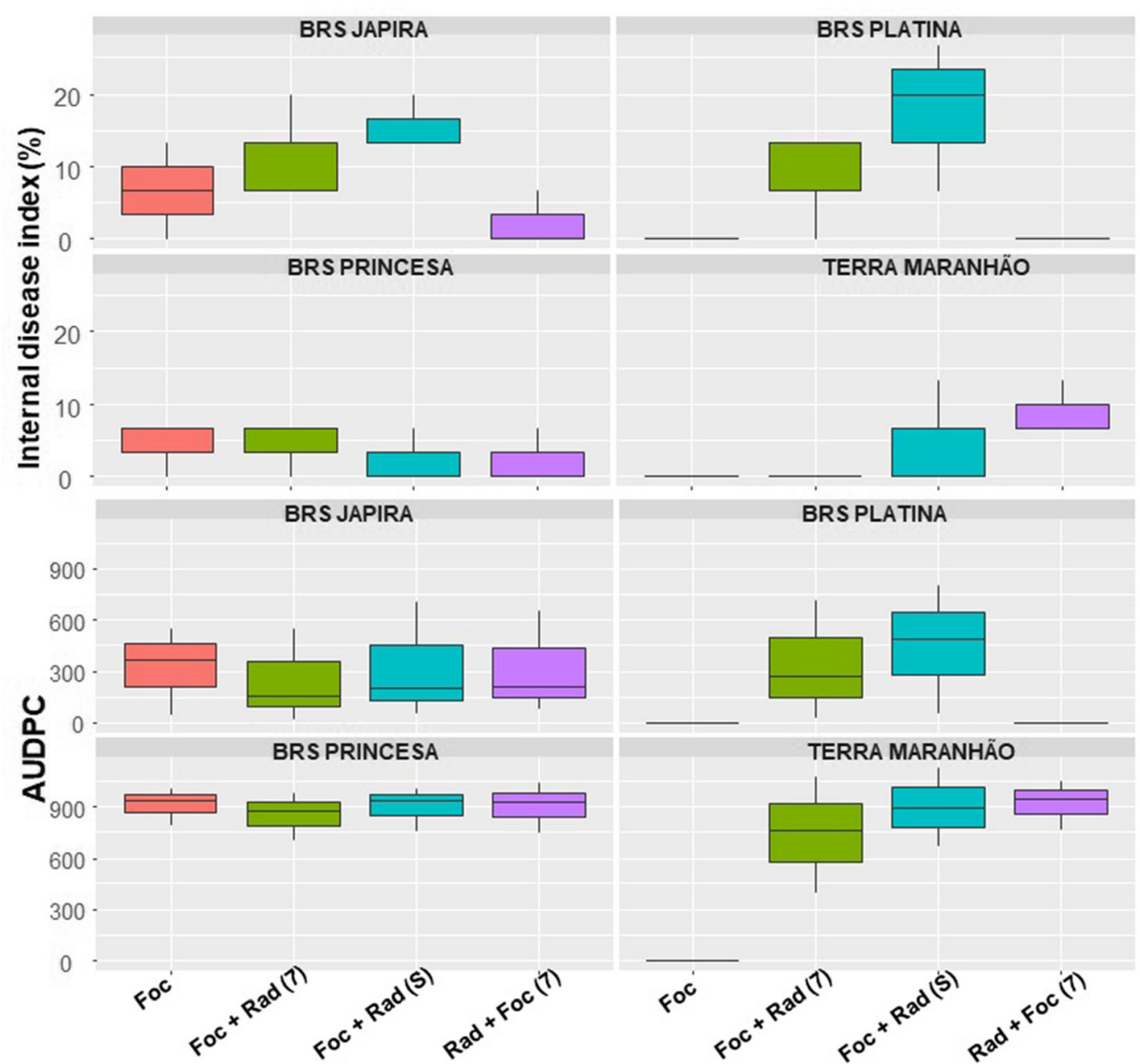

Fig. 2 Effects of the interaction between Fusarium oxysporum $\mathrm{f}$. sp. cubense (isolate 0801, race 1) and Radopholus similis in banana cultivars. Foc: Foc inoculated isolated; Foc + Rad (7): Foc inoculated in the planting and $R$. similis inoculated after 7 days;

functional roots in relation to that of other treatments. For Foc 0801, the treatment in which $R$. similis was inoculated singly had a greater reduction in the functional roots (Fig. 5).

Considering functional root reduction, 'BRS Princesa' had the highest percentage of reduction in functional roots in the treatment with simultaneous inoculation of Foc 0801 and $R$. similis (Fig. 5). For 'Terra Maranhão', Foc inoculation $7 \mathrm{~d}$ before that of $R$. similis and $R$. similis 7 days before that of Foc had the greatest reduction in the percentile of functional roots (Fig. 5). The percentage of the reduction of functional roots was similar in 'BRS Vitória', 'BRS Japira', and 'BRS Platina', and in all cultivars, the highest means were recorded
Foc + Rad (S): Foc and R. similis inoculated simultaneously; Rad + Foc (7): R. similis inoculated in the planting planting and Foc inoculated after 7 days. AUDPC: area under the disease progress curve

in the treatment in which $R$. similis was inoculated singly (Fig. 5).

Visualization of pathogen structures in the roots

For the controls without Foc inoculation, no internal symptoms or fungal structures were detected (Fig. 6 and Fig. 7). 'Prata-Anã' and 'Grande Naine' cultivars, inoculated only with the Foc 0801 isolate, showed abundant production of chlamydospores within the root tissue (Fig. $6 \mathrm{~g}$ and $\mathrm{m}$ ). For the Foc treatments $7 \mathrm{~d}$ before inoculation with $R$. similis and the simultaneous inoculation of both pathogens, the presence of hyphae and chlamydospores was observed (Fig. $6 \mathrm{~h}, \mathrm{n}, \mathrm{i}, \mathrm{o}$ ). In the 


\section{Correlation isolate 0801}

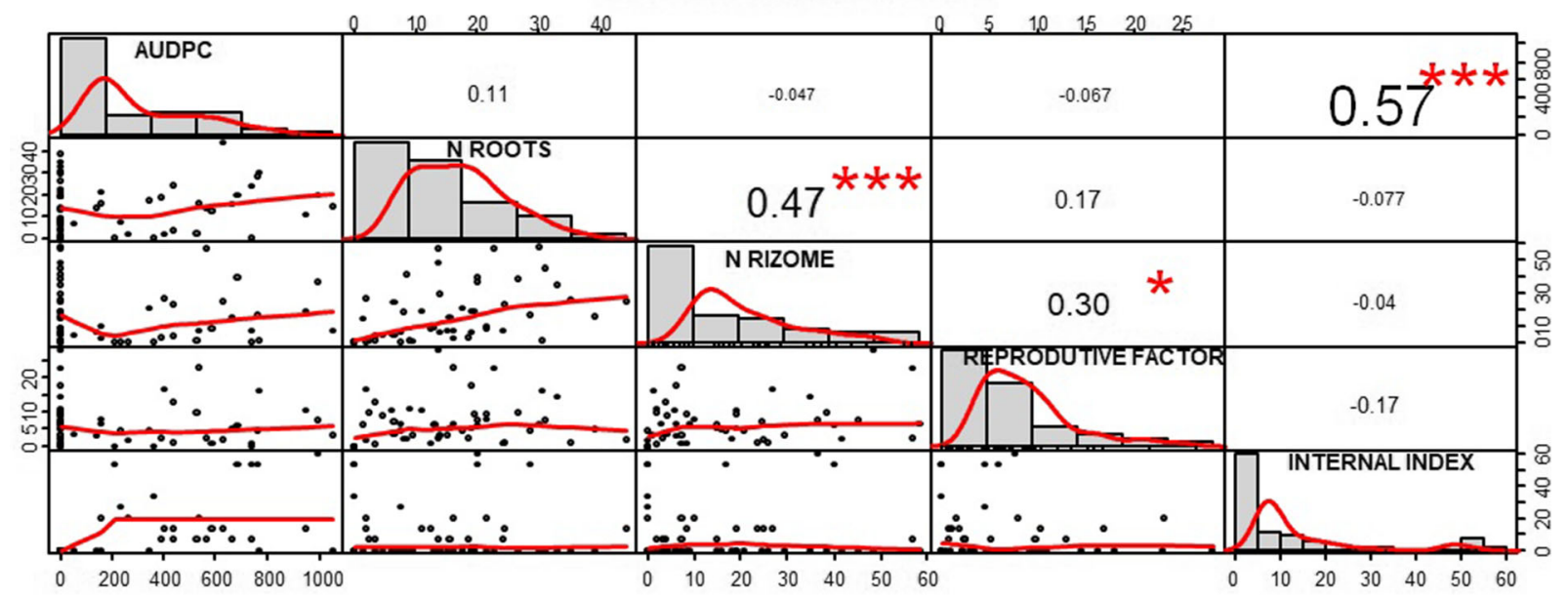

\section{Correlation isolate 218A}

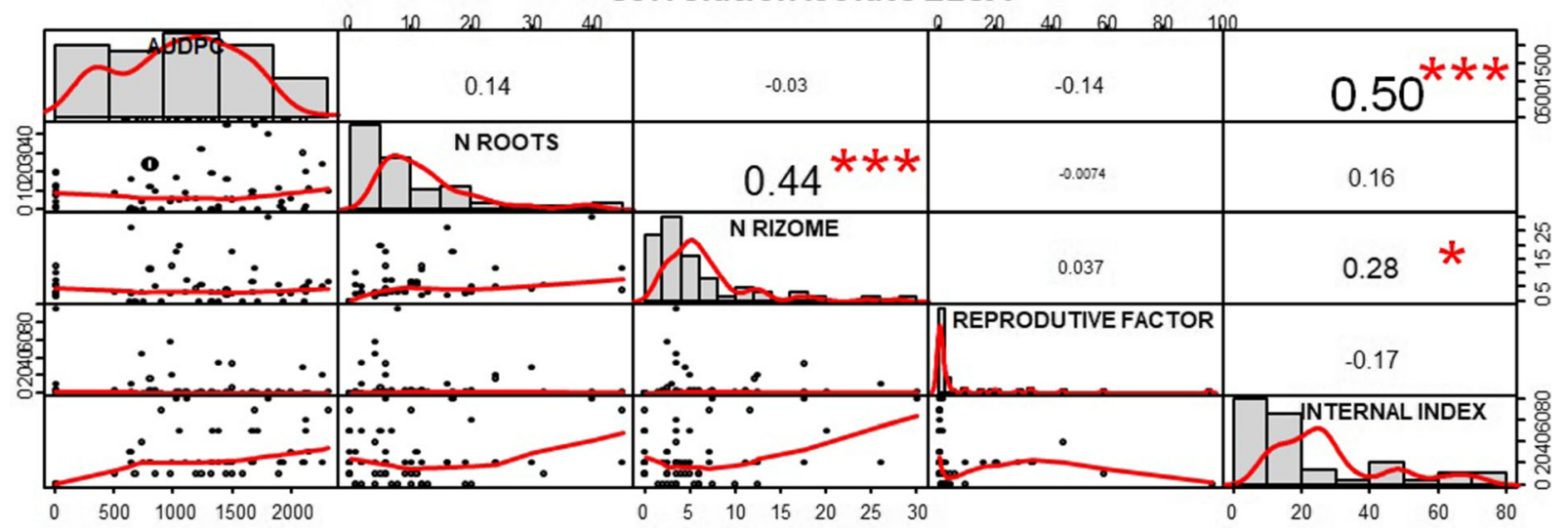

Fig. 3 Correlograms are shown for the parameters evaluated in the study of interaction between Fusarium oxysporum f. sp. cubense and Radopholus similis in banana cultivars: internal disease index (IDI), area under the disease progress curve (AUDPC), reproduction factor (RF), root necrosis (NRoot) and rhizome necrosis (NRhizome). The distribution of each variable is shown diagonally. At the bottoms of the diagonals, bivariate scatter plots with a fitted line are shown. At the tops of the diagonals, the Pearson correlation values plus the significance level as stars are displayed. *** $P<0.001$; ** $P<0.01 ; * P<0.05$
R. similis treatment 7 days prior to that of Foc, the presence of clustered chlamydospores was observed (Fig. 6p). Furthermore, for 'Grand Naine' it was possible to detect $R$. similis in plants inoculated with the nematode singly (Fig. 6q).

In relation to 'BRS Platina', based on the histological evaluation using light microscopy, there were no structures for Foc in the internal tissues (Fig. 6a). However, the presence of chlamydospores inside the tissues was noted for the Foc treatments 7 days before $R$. similis, in the simultaneous inoculation of both pathogens, and in R. similis inoculated 7 days prior to Foc, (Fig. 6b-d).
$R$. similis was observed in the tissues of plants that were inoculated only with the nematode (Fig. 6).

Hyphae and chlamydospores of Foc within the tissues could be observed in the tissues of all cultivars, except in the control treatment (Fig. 7f, s, r). For 'Grand Naine', the presence of grouped and chain of chlamydospores was observed for treatments with $R$. similis (Fig. $7 \mathrm{n}, \mathrm{o}, \mathrm{p}$ ). Additionally, the presence of $R$. similis was confirmed in the roots of the plants inoculated with $R$. similis only (Fig. 7q).

For 'Prata-Anã', the presence of Foc was confirmed by visualization of abundant production of chlamydospores and hyphae within the tissue in all treatments in 
a)

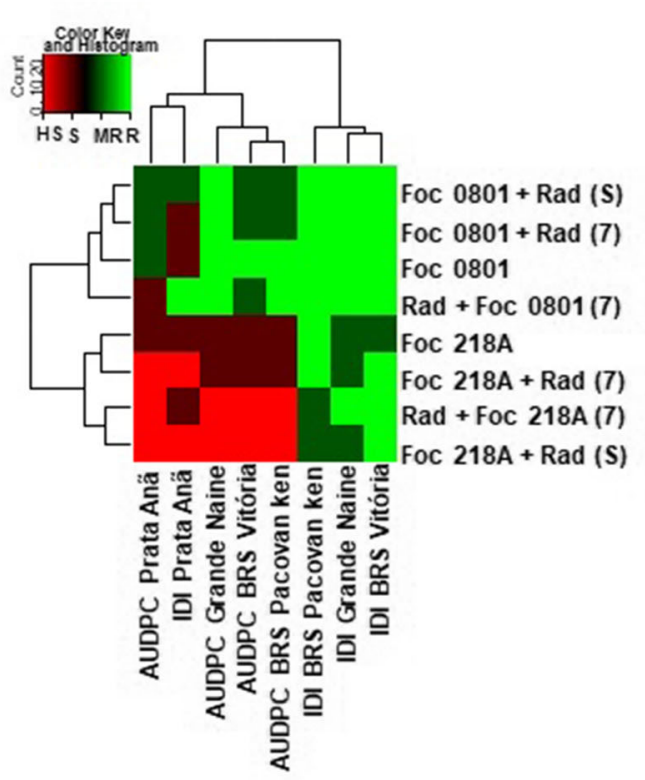

c)

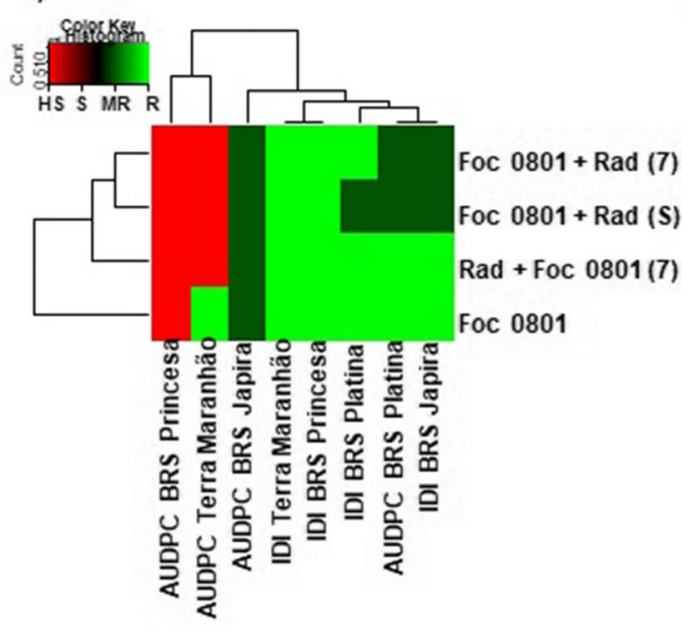

Fig. 4 Heat maps and cluster analysis of the interaction between Fusarium oxysporum f. sp. cubense (Foc) and Radopholus similis $(\mathrm{Rad})$ in banana cultivars. The cultivars are associated with different variables related to Foc (A) and Rad (B). Maps A and $\mathrm{B}$ show the result of the first experiment, in which two isolates of Foc (218A and 0801 race 1) were used. Maps C and D show the result of a second experiment, in which only one Foc isolate was used $(0801$, race 1$)$. The colors of the heat map reflect the data of the variables converted into a numerical matrix. $H S$ highly susceptible, $S$ susceptible, $R M$ Moderately susceptible and $R$

which Foc was inoculated (Fig. 7g, h, i, j). Treatments comprised by the concomitant inoculation of Foc and $R$. similis, and that of $R$. similis $7 \mathrm{~d}$ prior to Foc, exhibited b)

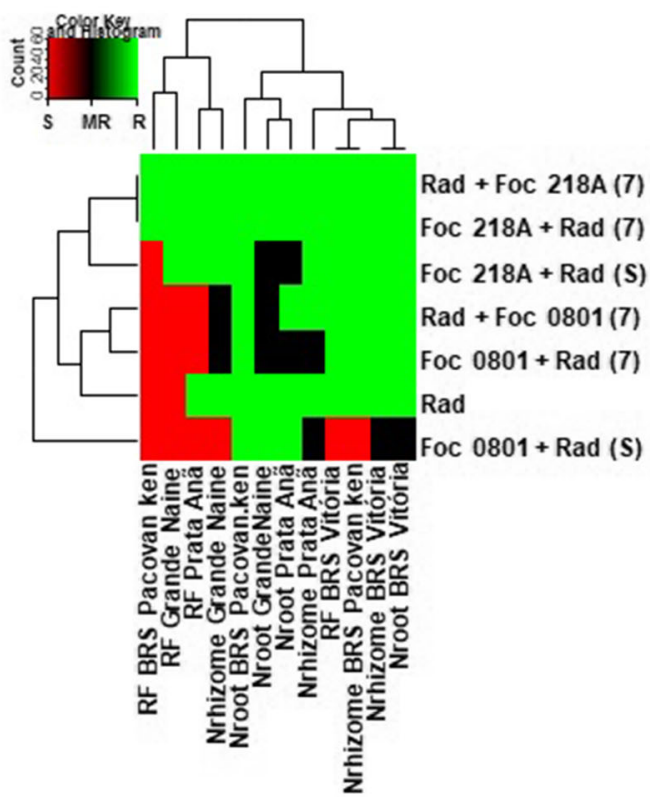

d)

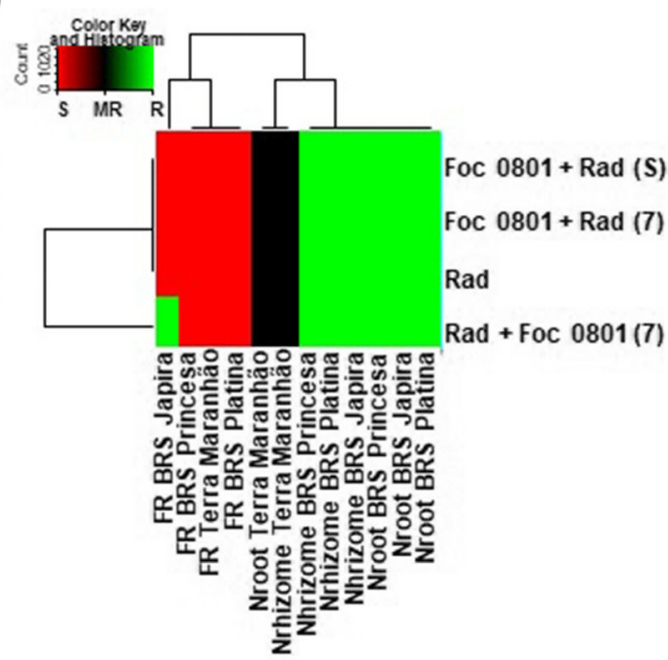

resistant. Description of variables: internal disease index (IDI), area under the disease progress curve (AUDPC), reproduction factor (RF), root necrosis (NRoot) and rhizome necrosis (NRhizome). Description of treatments: Foc: Foc inoculated isolated; Foc + Rad (7): Foc inoculated in the planting and R. similis inoculated after 7 days; Foc $+\operatorname{Rad}(\mathrm{S})$ : Foc and R. similis inoculated simultaneously; $\mathrm{Rad}+\mathrm{Foc}(7):$ R. similis inoculated in the planting planting; Foc inoculated after 7 days and Rad: $R$. similis inoculated isolated

large clustering of chlamydospores (Fig. 7i, j). Regarding the inoculation of 'Prata-Anã', there was no visualization of $R$. similis in the internal tissues (Fig. 7k). 


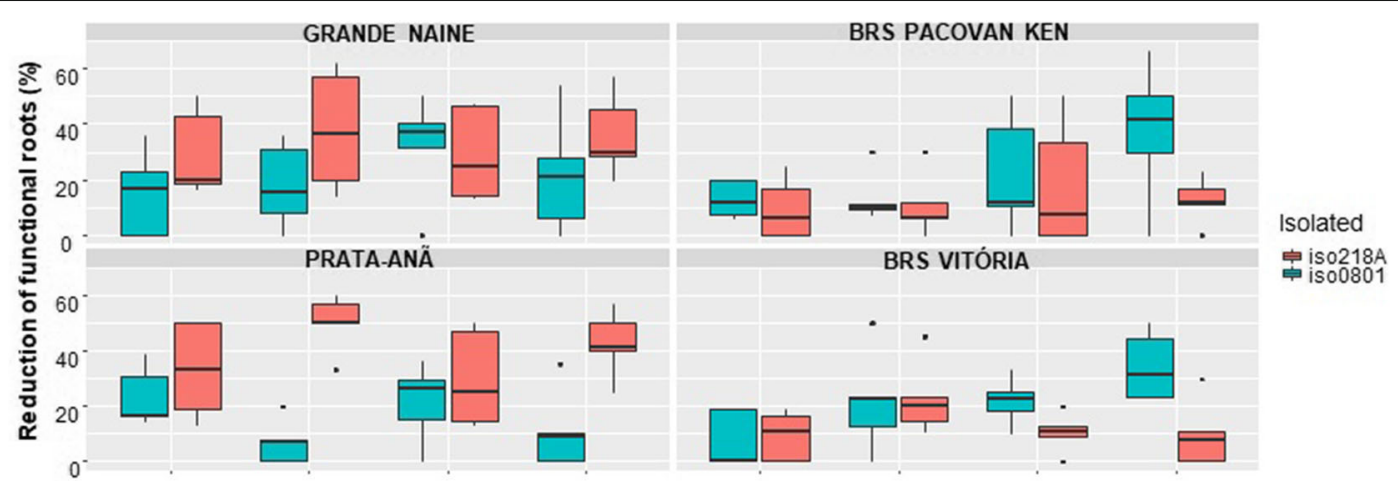

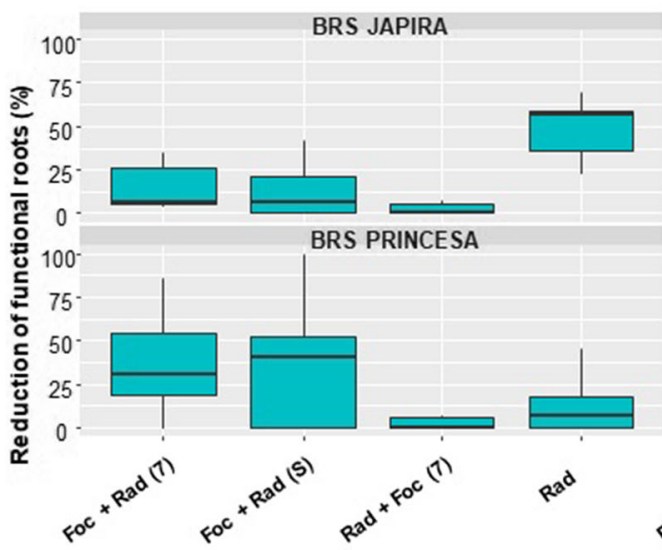

Fig. 5 Influence of different orders of inoculation of Fusarium oxysporum f. sp. cubense (Foc) and Radopholus similis (Rad) on the percentage reduction of functional roots of banana cultivars. Foc $+\operatorname{Rad}(7):$ Foc inoculated in the planting and $R$. similis

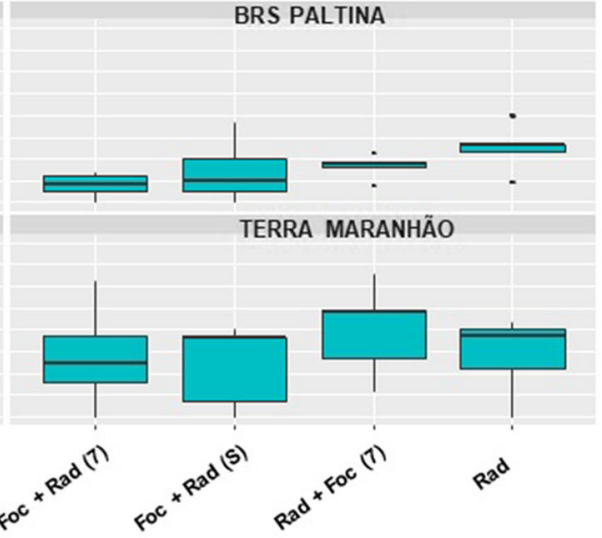

inoculated after 7 days; Foc $+\operatorname{Rad}(\mathrm{S})$ : Foc and $R$. similis inoculated simultaneously; Rad + Foc (7): R. similis inoculated in the planting planting and Foc inoculated after 7 days. Rad: $R$. similis inoculated isolated

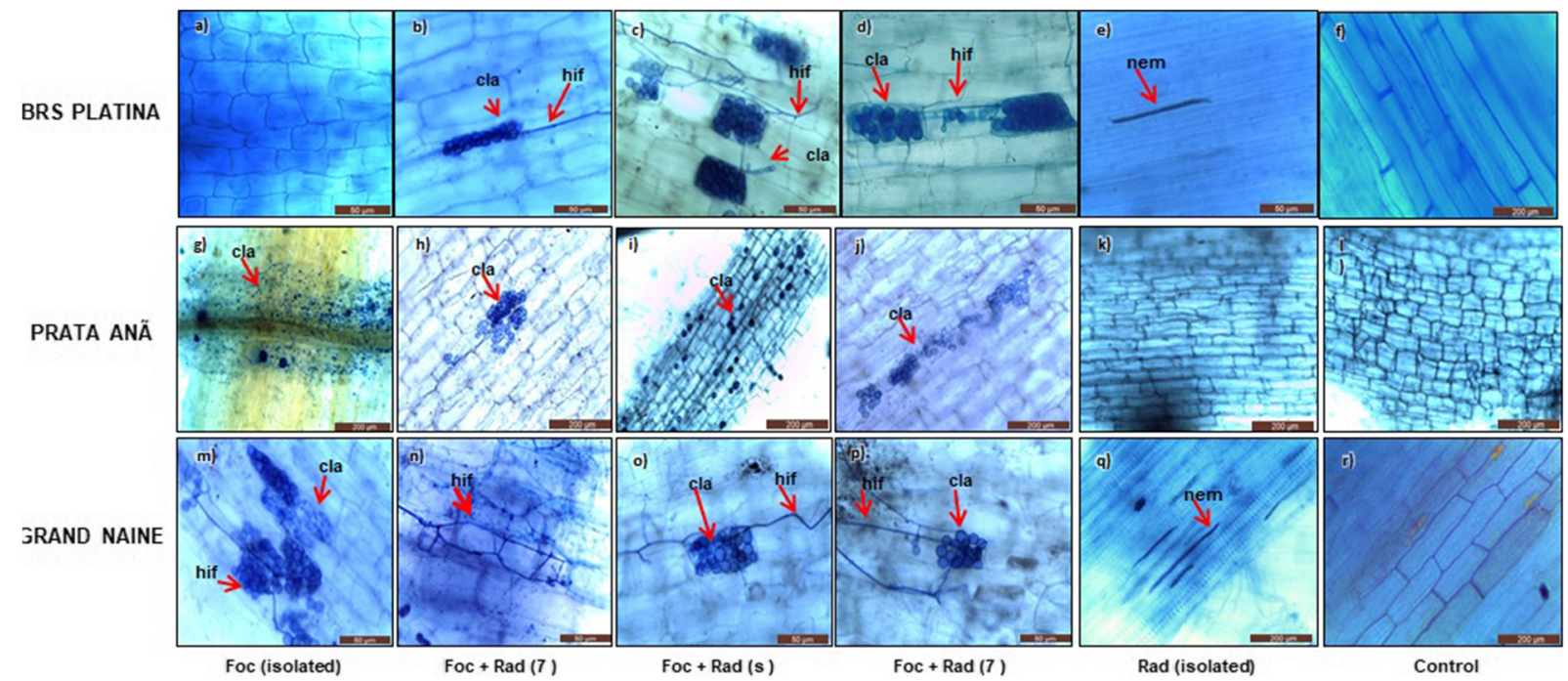

Fig. 6 Influence of different orders of inoculation of Radopholus similis (Rad) on the penetration of Fusarium oxysporum f. sp. cubense (Foc isolate 0801, race 1) in the root tissues of banana cultivars. Foc: Foc inoculated isolated; Foc $+\operatorname{Rad}(7)$ : Foc inoculated in the planting and R. similis inoculated after 7 days; Foc + Rad (S): Foc and R. similis inoculated simultaneously; Rad + Foc (7): R. similis inoculated in the planting planting and Foc inoculated after 7 days; Rad: $R$. similis inoculated isolated 


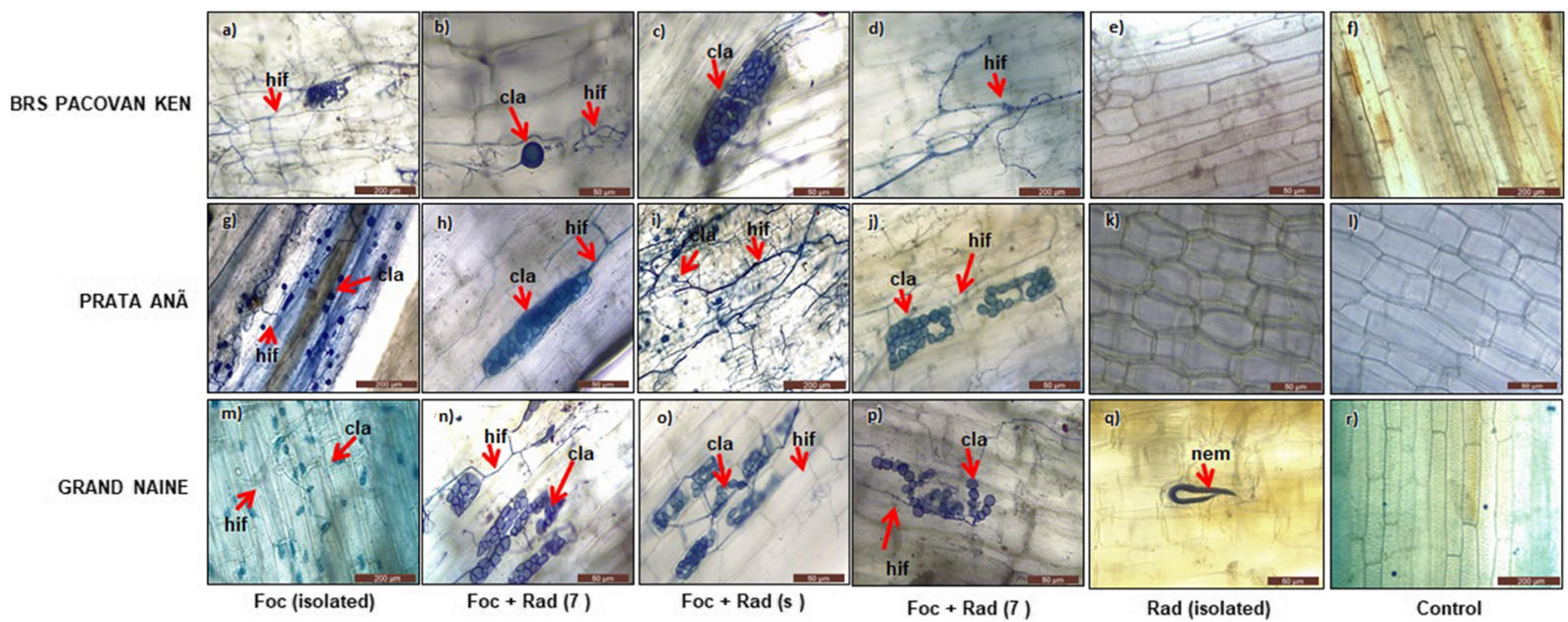

Fig. 7 Influence of different orders of inoculation of Radopholus similis (Rad) on the penetration of Fusarium oxysporum f. sp. cubense (Foc isolate 218A) in the root tissues of banana cultivars. Foc: Foc inoculated isolated; Foc $+\operatorname{Rad}(7)$ : Foc

\section{Discussion}

In this study, the simultaneous or alternating inoculation at a 7 days interval of Foc and $R$. similis in banana cultivars resistant to FW allowed the verification of the effects of the interaction on the resistance response of the banana cultivars to FW using two Foc isolates differing in virulence characteristics. It was therefore confirmed that the dietary habits and life cycle of plant parasitic nematodes are fundamental to their role in the predisposition of a plant to infection (Simão et al. 2010; Piedrahita, 2011; Ali et al. 2017).

Thus, the results showed that the type of resistance experienced by the cultivars in relation to Foc, as well as the virulence of the isolate are fundamental aspects in the occurrence of resistance or lack thereof to FW when it interacts with $R$. similis. Thus, the hypothesis of changes in the resistance behavior as a result of the interaction between Foc 0801 and $R$. similis was experienced only for the cultivars 'BRS Pacovan Ken', 'BRS Platina', 'BRS Vitória', and 'Terra Maranhão', whereas different interactions of Foc with $R$. similis may lead to the quantitative type of resistance, where it was possible to measure different levels of severity in treatments of interactions between the pathogens.

In some studies, it has been verified that many mechanisms of penetration and infection of $R$. similis and other migratory endoparasite nematodes are related to the breaking of preformed barriers, such as lignification of the cell wall or presence of cellulose and inoculated in the planting and $R$. similis inoculated after 7 days; Foc + Rad (S): Foc and R. similis inoculated simultaneously; Rad + Foc (7): R. similis inoculated in the planting planting and Foc inoculated after 7 days; Rad: $R$. similis inoculated isolated

hemicellulose (Haegeman et al. 2008). These aspects could be linked with the changes in the resistance behavior of some banana cultivars because of the interaction between the nematode and Foc. This is probably because the rapid penetration of $R$. similis into the roots and the secretion of enzimes cellulase and endoxylanase can lead to the degradation of hemicellulose from the plant cell walls (Haegeman et al. 2010).

Through the staining of Foc and $R$. similis structures with trypan blue inside the tissue, the influence of $R$. similis on the penetration of Foc in tissues of resistant and susceptible cultivars was confirmed (Figs. 6 and 7). According to Costa (2013), the type of resistance response involved in the interaction between the isolate Foc 0801 and the cultivar BRS Platina is based on cell wall modifications, such as the formation of a healing zone, the presence of tylosis, oxalate crystals, and lignification. Therefore, the data presented here suggest that the penetration of $R$. similis causes damage to these preformed barriers, allowing the penetration and colonization of the pathogen within the tissues and consequent manifestation of symptoms in plants with this type of resistance mechanisms.

When using the isolate $218 \mathrm{~A}$, the qualitative resistance reaction of bananas cultivars to Foc was not dependent on the interaction with $R$. similis and Foc because the virulence was observed even for banana cultivars belonging to the Cavendish group ('Grande Naine') (Fig. 1). However, a quantitative aspect was observed because there was an increase in disease severity as measured by root necrosis 
and internal necrosis (Supplementary Fig. 2). In this case, the interaction with $R$. similis in most cultivars was related to the increase in the severity of symptoms, as suggested in the hypothesis for this study. In previous studies, an increase in the severity of FW was also observed because of the concomitant presence of these pathogens in cultivars susceptible to Foc (Somu et al. 2013; Dinesh et al. 2014).

In the evaluation of the cultivar 'Grande Naine', staining the roots demonstrated that Foc 0801 penetrated the tissues of the plants, even in the treatments that lacked $R$. similis, but there was no manifestation of FW symptoms for any of the treatments tested (Figs. 1 and 6). The reaction differences expressed distinctions in the mechanisms inherent to the resistance of the cultivars to FW. Thus, the resistance of the Cavendish cultivars is not only related to Foc penetration. These arguments were confirmed by Li et al. (2013), who found the existence of different regulatory proteins that are related to diverse responses of banana cultivars to Foc, especially cultivars resistant to Foc TR4. According to studies by Li et al. (2012), differentially expressed genes and signaling pathways revealed the defense system of Cavendish bananas could be very complex.

These arguments explain the fact that the high susceptibility of the cultivar 'Grande Naine' to R. similis does not interfere with the behavior of the cultivar in relation to Foc 0801 (Race 1). However, when using the $218 \mathrm{~A}$ isolate the data indicated a greater ability of Foc to cause disease in more cultivars, including the 'Grande Naine' cultivar (subgroup Cavendish) that is not affected by FW in Brazil (Fig. 1 and Supplementary Fig. 2). These results are related to the virulence of the $218 \mathrm{~A}$ isolate. Costa et al. (2015) demonstrated the evolutionary potential of Foc in Brazil, which resulted in high genetic variability that gave rise to more virulent pathogens capable of infecting resistant cultivars.

For the cultivar 'BRS Princesa', the Foc 0801 isolate data showed there was no interference for the different orders of inoculation of $R$. similis and Foc on the behavior of the cultivar in relation to Foc, and that all treatments had low indices of internal, which allowed it to be grouped as resistant. According to Costa et al. (2015), the 'BRS Princesa' cultivar could exhibit quantitative resistance, although there are no reports of infections of this cultivar in the field. Therefore, the results presented here could be related to the fact that quantitative resistance was considered more durable than qualitative resistance, as well as that this type of resistance reduces the multiplication of the pathogen, colonization, and severity of symptoms (Pilet-Nayel et al. 2017), making it efficient against both pathogens.

In addition, it was clear in this study that cultivars that behaved as resistant to isolated $R$. similis had altered responses regarding its interaction with Foc 0801 and 218A and became highly susceptible (Fig. 4). Thus, the increase in the intensity of FW symptoms and increased reproductive factor or necrosis of roots and rhizomes in banana plants in relation to the interaction of $R$. similis with more virulent isolates or variants of the pathogen could decrease plant life. In these cases, the incubation period of Foc was reduced and there was a rapid manifestation of symptoms and plant death, as well as a reduction in plant productivity because plant development parameters were more affected by the joint action. This fact was related to the increase in necrotic areas in the root and rhizome tissues that reduced water and nutrient absorption, debilitated development, reduced production, and increased the time between harvests (Gowen and Quénéhervé, 1990).

Thus, it was concluded that the nematode $R$. similis has the potential to alter the defense responses of banana cultivars to FW, which can lead to unexpected results in the field. For this reason, alternatives for controlling and managing $R$. similis populations are needed in cultivation areas as a control strategy for FW. The results indicated that both alternating and simultaneous inoculation of the pathogens have effects on the incidence of FW and development parameters of the plants. This suggests that controlling populations of the nematode needs to be conducted both before planting banana cultivars and while monitoring plant growth to avoid the introduction of $R$. similis in the cultivation area.

In this study, for screening of banana cultivars for resistance to $R$. similis were used part of the methods indicated by Speijer and De Waele (1997). According to these authors the evaluation/interpretation of the data obtained during the nematode resistance/ tolerance screening should be based on a combination of nematode reproduction data and host plant response data including at least: number of nematodes in the roots, percentage of dead roots and root necrosis indexmby preference taken at different moments during plant growth (for instance early plant growth, e.g. 2 months after planting, at flowering and at harvesting). The combination of the data will give a reliable indication that the genotype is resistant or susceptible, tolerant or sensitive (Speijer and De Waele 1997). 
Although studies consider that resistance to plant parasitic nematodes is commonly defined as a reduction in nematode reproduction, nematode extraction and counting processes are not accurate methods. The symptoms and levels of damage (necrosis in the rhizome, aspects of productivity and development of the plant) could be more accurate and more efficient methods in discriminating banana genotypes resistant to $R$. similis. Therefore, from this study, it is considered that in situations of interaction between Foc and $R$. similis, the set of efficient and easy methods to discriminate banana genotypes resistant to both pathogens may start from symptom measurement techniques, with scales of notes, followed by field evaluations that include agronomic characterization of plants.

In general, the data generated in this study have relevant implications for banana breeding programs, because screening techniques for resistance to WF may include screening techniques related to resistance to $R$. similis. This would allow for greater precision and greater reliability as to the behavior of cultivars in the field. Therefore, as part of the routine screening for resistance, in addition to inoculations in the greenhouse, the field test should be performed. The data herein provide new perspectives to understand the mechanisms involved in the interaction between Foc and $R$. similis in banana plants and will be important in management and control strategies for FW. In addition, further studies should be carried out to verify the response of cultivars to the interaction of Foc and $R$. similis at histological and/or genetic levels.

Acknowledgments The authors thank the Coordenação de Aperfeiçoamento Pessoal de Nível Superior (CAPES), Breeding Better Bananas/ IITA/Bill and Melinda Gates Foundation project for the financial support to conduct the study and Embrapa Mandioca e Fruticultura for offering the infrastructure.

Funding The Coordenação de Aperfeiçoamento Pessoal de Nível Superior (CAPES).

\section{Compliance with ethical standards}

Conflict of interest The authors declare that they have no conflicts of interest.

Open Access This article is licensed under a Creative Commons Attribution 4.0 International License, which permits use, sharing, adaptation, distribution and reproduction in any medium or format, as long as you give appropriate credit to the original author(s) and the source, provide a link to the Creative Commons licence, and indicate if changes were made. The images or other third party material in this article are included in the article's Creative Commons licence, unless indicated otherwise in a credit line to the material. If material is not included in the article's Creative Commons licence and your intended use is not permitted by statutory regulation or exceeds the permitted use, you will need to obtain permission directly from the copyright holder. To view a copy of this licence, visit http://creativecommons.org/licenses/by/4.0/.

\section{References}

Ali, M. A., Azeem, F., Li, H., \& Bohlmann, H. (2017). Smart parasitic nematodes use multifaceted strategies to parasitize plants. Frontiers in Plant Science, 8, 1699. https://doi. org/10.3389/fpls.2017.01699.

Amorim, E. P., dos Serejo, J. A. S., Amorim, V. B. O., \& Silva, S. O. (2016). Melhoramento genético. In C. F. Ferreira, S. O. Silva, E. P. Amorim, \& J. A. Santos-Serejo (Eds.), (1a ed., pp. 171-200). O agronegócio da banana: Embrapa.

Bridge J. (1988). Plant nematode pests of banana in East Africa with particular reference to Tanzania. Nematodes and the borer weevil in bananas. Present status of research and outlook, 35-39.

Buddenhagen I.W. (2009). Understanding strain diversity in Fusarium oxysporum f. sp. cubense and history of introduction of tropical race 4 to better manage banana production. In: Jones, D.; Van Den Bergh, I. (Ed.). Proceedings of the International Symposium on Recent Advances in Banana Crop Protection for Sustainable Production and Improved Livelihoods, White River, South Africa. ISHS Acta Horticulturae, 828, 193-204.

Campbell, C. L., \& Madden, L. V. (1990). Introduction to plant disease epidemiology (532 p). New York: Wiley.

Coolen W.A., D'Herde C.J. (1972). A method for the quantitative extraction of nematodes from plant tissue. Ghent, State Nematology and Entomology Research 379, Station 77.

Costa J.L. (2013). Estudos histológicos e moleculares da interação Musa spp. x Fusarium oxysporum f. sp. cubense. Piracicaba, São Paulo: Universidade de São Paulo, Dissertação (Mestrado em ciências).

Costa, S. N., Bragança, C. A. D., Ribeiro, L. R., Amorim, E. P., Oliveira, S. A. S., Dita, M. A., \& Laranjeira, F. F. (2015). Haddad F. Plant Pathology: Genetic structure of Fusarium oxysporum $\mathrm{f}$. sp. cubense in different regions from Brazil. https://doi.org/10.1111/ppa.12242.

Davis, E. L., Hussey, R. S., Baum, T. J., Bakker, J., Schots, A., Rosso, M. N., \& Abad, P. (2000). Nematode parasitism genes. Annual Review of Phytopathology, 38(1), 365-396. https://doi.org/10.1146/annurev.phyto.38.1.365.

Davis, E. L., Hussey, R. S., \& Baum, T. J. (2004). Getting to the roots of parasitism by nematodes. Trends in Parasitology, 20(3), 134-141. https://doi.org/10.1016/j.pt.2004.01.005.

Dinesh, B. M., Ravichandra, N. G., Reddy, B. M., \& Somasekhara, Y. M. (2014). Interactions between Radopholus similis and Fusarium oxysporum f. sp. cubense causing wilt complex on Banana. International Journal of Advanced Research, Indore, 2, 976-985.

Dita, M., Barquero, M., Heck, D., Mizubuti, E. S., \& Staver, C. P. (2018). Fusarium wilt of banana: Current knowledge on epidemiology and research needs toward sustainable disease 
management. Frontiers in Plant Science, 9. https://doi. org/10.3389/fpls.2018.01468.

Dita, M. A., Pérez, V. L., \& Martinez De La Parte, E. (2014). Inoculation of Fusarium oxysporum $\mathrm{f}$. sp. cubense causal agent of fusarium wilt in banana. Technical Manual: Prevention and diagnostic of Fusarium Wilt of banana caused by Fusarium oxysporum f. sp. cubense. Tropical Race, 4, 55-58.

García-Bastidas, F. A., Quintero-Vargas, J. C., Ayala-Vasquez, M., Schermer, T., Seidl, M. F., Santos-Paiva, M., et al. (2019). First report of fusarium wilt tropical race 4 in cavendish bananas caused by fusarium odoratissimum in Colombia. Plant Disiese. https://doi.org/10.1094/PDIS-0919-1922-PDN.

Gowen, S. P., \& Quénéhervé, P. (1990). Nematode parasites of bananas and abaca. In M. Luc, R. A. Sikora, \& J. Bridge (Eds.), Plant parasitic nematodes in subtropical and tropical agriculture (pp. 431-460). Wallingford: C.A.B. International.

Ghag, S. B., Shekhawat, U. K., \& Ganapathi, T. R. (2015). Fusarium wilt of banana: Biology, epidemiology and management. International Journal of Pest Management, 61(3), 250-263. https://doi.org/10.1080/09670874.2015.1043972.

Haddad, F., Rocha, L. S., Soares, A. C. F., Martins, I. P. S., Teixeira, L. A. J., Staver, C., \& Dita, M. (2018). Management of Fusarium wilt of bananas in Minas Gerais, Brazil. Acta Horticulturae, 1, 137-146. https://doi. org/10.17660/ActaHortic.2018.1196.16.

Haegeman, A., Elsen, A., De Waele, D., \& Gheysen, G. (2010). Emerging molecular knowledge on Radopholus similis, an important nematode pest of banana. Molecular Plant Pathology, 11(3), 315-323. https://doi.org/10.1111/j.13643703.2010.00614.x.

Haegeman, A., Jacob, J., Vanholme, B., Kyndt, T., \& Gheysen, G. (2008). A family of GHF5 endo-1, 4-beta-glucanases in the migratory plant-parasitic nematode Radopholus similis. Plant Pathology, 57, 581-590. https://doi.org/10.1111/j.13653059.2007.01814.x.

Hartman, J. B., Vuylsteke, D., Speijer, P. R., Ssango, F., Coyne, D. L., \& De Waele, D. (2010). Measurement of the field response of Musa genotypes to Radopholus similis and Helicotylenchus multicinctus and the implications for nematode resistance breeding. Euphytica, 172(1), 139-148. https://doi.org/10.1007/s10681-009-0104-4.

Hussey, R., Davis, E., \& Baum, T. (2002). Secrets in secretions: Genes that control nematode parasitism of plants. Brazilian Journal of Plant Physiology, 14, 183-194. https://doi. org/10.1590/S1677-04202002000300002.

Jenkins, W. (1964). A rapid centrifugal-flotation technique for separating nematodes from soil. Plant disease reporter, $48(9), 692$.

Jones, J. T., Haegeman, A., Danchin, E. G., Gaur, H. S., Helder, J., Jones, M. G. K., Kikuchi, T., Manzanilla-López, R., Palomares-Rius, J. E., Wesemael, W. M. L., \& Perry, R. N. (2013). Top 10 plant-parasitic nematodes in molecular plant pathology. Molecular Plant Pathology, 14(9), 946-961. https://doi.org/10.1111/mpp.12057.

Li, C. Y., Deng, G. M., Yang, J., Viljoen, A., Jin, Y., Kuang, R. B., Zuo, C. W., Lv, Z. C., Yang, Q. S., Sheng, O., Wei, Y. R., Hu, C. H., Dong, T., \& Wei, Y. R. (2012). Transcriptome profiling of resistant and susceptible Cavendish banana roots following inoculation with Fusarium oxysporum f. sp. cubense tropical race 4. BMC Genomics, 13(1), 374. https://doi.org/10.1186/1471-2164-13-374.

Li, X., Bai, T., Li, Y., Ruan, X., \& Li, H. (2013). Proteomic analysis of Fusarium oxysporum f. sp. cubense tropical race 4-inoculated response to Fusarium wilts in the banana root cells. Proteome Science, 11(1), 41.

Mckinney, H. H. (1923). Influence of soil, temperature and moisture on infection of wheat seedlings by Helminthosporium sativum. Journal of Agricultural Research, Washington, 26, 195-217. https://doi.org/10.1186/1477-5956-11-41.

Phillips, J. M., \& Hayman, D. S. (1970). Improved procedures for clearing roots and staining parasitic and vesicular-arbuscular mycorrhizal fungi for rapid assessment of infection. Transactions of the British Mycological Society, 55, 158161. https://doi.org/10.1016/S0007-1536(70)80110-3.

Piedrahita, O. A. G. (2011). El nematodo barrenador (Radopholus similis [Cobb] Thorne) del banano y plátano. Revista Luna Azúl, 32, 137-153.

Pilet-Nayel, M. L., Moury, B., Caffier, V., Montarry, J., Kerlan, M. C., Fournet, S., Durel, C. E., \& Delourme, R. (2017). Quantitative resistance to plant pathogens in pyramiding strategies for durable crop protection. Frontiers in Plant Science, 8, 1838. https://doi.org/10.3389/fpls.2017.01838.

Puhalla, J. E. (1985). Classification of strains of Fusarium oxysporum on the basis of vegetative compatibility. Canadian Journal of Biology, 63, 179-183. https://doi. org/10.1139/b85-020.

Ploetz, R. C. (2015). Fusarium wilt of banana. Phytopathology, 105, 1512-1521. https://doi.org/10.1094/PHYTO-04-150101-RVW.

Ploetz, R. C. (2006). Fusarium wilt of banana is caused by several pathogens referred to as Fusarium oxysporum f. sp. cubense. Phytopathology, 96, 653-656. https://doi.org/10.1094 /PHYTO-96-0653.

Ploetz R.C. (1990). Current status of Fusarium wilt of banana (Panama disease) in the western hemisphere. In Proceedings of the Interamerican Society for Tropical Horticulture (Vol. 34, pp. 169-176).

R Development Core Team. (2017). R: A language and environment for statistical computing R Foundation for Statistical Computing, Vienna, Austria (2017) ISBN 3-900051-07-0, URL: http://www.R-project.org/.

Rebouças, T. A., Haddad, F., Ferreira, C. F., De Oliveira, S. A. S., Da Silva Ledo, C. A., \& Amorim, E. P. (2018). Identification of banana genotypes resistant to Fusarium wilt race 1 under field and greenhouse conditions. Scientia Horticulturae, Maio, 1-6. https://doi.org/10.1016/j.scienta.2018.04.037.

Ribeiro, L. R., Oliveira, S. A. S. D., Amorim, E. P., Serejo, J. A. S., \& Haddad, F. (2018). Sources of resistance to Fusarium oxysporum f. sp. cubense in banana germplasm. Revista Brasileira de Fruticultura, 40(1). https://doi.org/10.1590 /0100-29452018202.

Rocha, L. S., Santana, R. F., Soares, A. C. F., \& Haddad, F. (2018). Reaction of banana cultivars to the Meloidogyne javanica X Fusarium oxysporum f. sp. cubense complex. Revista Caatinga, 31, 572-583. https://doi.org/10.1590 /1983-21252018v31n305rc.

Sankar, C., Soorianathasundaram, K., Kumar, N., Karunakaran, G., \& Sivakumar, M. (2017). Induction of resistant to Radopholus similis and Defence related mechanism in susceptible and resistance Banana hybrids infected with 
Radopholus similis. International Journal of Current Microbiology and Applied Sciences, 6(4), 1668-1684. https://doi.org/10.20546/ijcmas.2017.604.202.

Shapiro, S. S., \& Wilk, M. B. (1965). An analysis of variance test for normality (complete samples). Biometrika, 52(3/4), 591611 https://www.jstor.org/stable/2333709.

Silva, S. D. O., Matos, A. D., Cordeiro, Z. J. M., Lima, M. J. C., \& Amorim, E. P. (2011). Avaliação de genótipos tetraploides de bananeira cultivados em área infestada pelo agente causal do mal-do-Panamá. Revista Brasileira de Fruticultura, Jaboticabal, 33, 125-132.

Simão, G., Orsini, I. P., Sumida, C. H., Homechin, M., Santiago, D. C., \& Cirino, V. M. (2010). Reaction of cultivars and lines of bean in relation to Meloidogyne javanica and Fusarium oxysporum f. sp. phaseoli. Ciência Rural, 40, 1003-1008. https://doi.org/10.1590/S0103-84782010000500001.
Somu, R., Thammaiah, N., Devappa, V., Kulkarni, M. S., \& Swamy, G. S. K. (2013). Interaction studies of Fusarium oxysporum f. sp. cubense with burrowing nematode (Radopholus similis). International Journal of Plant Protection, 6, 70-72.

Speijer P.R., De Waele D. (1997). Screening of Musa germplasm for resistance and tolerance to nematodes (Vol. 1). IITA.

Stover, R. H. (1962). Fusarial wilt (Panama disease) of bananas and other Musa species. Kew: Commonwealth Mycological Inst.

Velame, K. V. C. (2017). Genes de bananeira candidatos à resistência a murcha de Fusarium e caracterização da agressividade e virulência do fungo. Feira de Santana: Universidade Estadual de Feira de Santana, Tese (Doutorado em Biotecnologia). 